\title{
Whole-body vibration as a modality for the rehabilitation of peripheral neuropathies: implications for cancer survivors suffering from chemotherapy-induced peripheral neuropathy
}

\author{
Anna L.J. Verhulst, ${ }^{1}$ Hans H.C.M. Savelberg, ${ }^{2}$ Gerard Vreugdenhil,, ${ }^{3,4}$ Massimo Mischi, ${ }^{5}$ \\ Goof Schep ${ }^{6}$ \\ ${ }^{1}$ Faculty of Health, Medicine and Life Sciences, Maastricht University, Maastricht; \\ ${ }^{2}$ Department of Human Movement Sciences, Maastricht University Maastricht; \\ ${ }^{3}$ Department of Medical Oncology, Máxima Medical Centre, Veldhoven; ${ }^{4}$ Department of \\ Medical Oncology, Maastricht University Medical Centre, Maastricht; ${ }^{5}$ Department of \\ Electrical Engineering, Eindhoven University of Technology, Eindhoven; ${ }^{6}$ Department of \\ Sports Medicine, Máxima Medical Centre, Veldhoven, The Netherlands
}

\begin{abstract}
The objective was to study the effect of whole-body vibration (WBV) on strength, balance and pain in patients with peripheral neuropathies and to consider its significance for the rehabilitation of patients suffering from chemotherapy-induced peripheral neuropathy (CIPN). Using a broad search strategy, PubMed was searched for clinical trials on WBV interventions aimed at improving strength, balance or pain in patients with peripheral neuropathies, which were published in English until $5^{\text {th }}$ June 2014. The search was performed by the first author and generated a total of 505 results, which yielded 5 articles that met the inclusion criteria, being studies: i) published in English; ii) involving adult human subjects' peripheral neuropathies; iii) evaluating the effect of WBV as a therapeutic intervention; and iv) reporting findings for at least one of the following outcomes: strength, bal-
\end{abstract}

Correspondence: Anna L.J. Verhulst, Pisanostraat 588, 5623 CS, Eindhoven, The Netherlands.

Tel.: +316.14020649 - Fax: +3140.888 .82 .20 .

E-mail: a.verhulst@student.maastrichtuniversity.nl

Key words: chemotherapy-induced peripheral neuropathy, whole body vibration, cancer rehabilitation.

Contributions: AV, data collection, data analysis, manuscript writing; HS, data analysis, manuscript writing, manuscript review; MM, GV, manuscript writing, manuscript review; GS, manuscript review.

Conflicts of interests: authors declare no potential conflicts of interests.

Received for publication: 24 August 2014.

Revision received: 12 December 2014.

Accepted for publication: 17 December 2014.

This work is licensed under a Creative Commons Attribution NonCommercial 3.0 License (CC BY-NC 3.0).

(C) Copyright A.L.J. Verhulst et al., 2015

Licensee PAGEPress, Italy

Oncology Reviews 2015; 9:263

doi:10.4081/oncol.2015.263 ance or pain. Methodological quality of included studies was assessed independently by first and second author, using the physiotherapy evidence database scale. The overall methodological quality of included studies was low. Two studies found a beneficial effect of WBV on neuropathic pain, but another study failed to find the same effect. One study found significant improvements in both muscle strength and balance, while another study found improvements only in some, but not all, of the applied tests to measure muscle strength and balance. The results of this literature search suggest insufficient evidence to assess the effectiveness for the effects of WBV on neuropathic pain, muscle strength and balance in patients with peripheral neuropathies. More high-quality trials are needed to guide the optimization of rehabilitation programs for cancer survivors with CIPN in particular.

\section{Introduction}

A common side effect of cancer treatment with chemotherapy is chemotherapy-induced peripheral neuropathy (CIPN). This neuropathy often occurs in treatment with platinum agents, a number of antimitotic drugs (taxanes, vinca alkaloids and thalidomide) and bortezomib. ${ }^{1}$ These neurotoxic agents target the nervous system at different sites, including the dorsal root ganglions, and the microtubules, ion channels and blood vessels of peripheral axons. ${ }^{2}$ Symptoms are dose-dependent, and mostly sensory (e.g. pain, loss of sensibility) but they can also manifest as motor weakness or autonomic dysfunction. ${ }^{3,4}$ The symptoms of the peripheral nervous system are related to the components affected. Damage of small fibers causes pain, hyperesthesia and loss of pain and temperature senses, while injury to large fibers produces loss of vibration sense, loss of proprioception and muscle weakness. ${ }^{5}$ Currently available pharmacological agents to prevent or treat CIPN have not been proven conclusively effective or have shown only minor effects. ${ }^{3,6-10}$ The most recent clinical practice guideline of the American Society of Clinical Oncology states that no agents are recommended for the prevention of CIPN and that best available data only support a moderate recommendation for treatment with duloxetine. ${ }^{10}$ Since CIPN symptoms can persist for years after onset, they may have a significantly negative long-term effect on daily activities and quality of life in cancer survivors. ${ }^{11-13}$ Furthermore, CIPN is a major dose-limiting factor in cancer therapy and can even be a reason to terminate treatment prematurely, compromising patient survival. ${ }^{7}$ 
Finally, due to considerable progress in cancer treatment and survival rates, the population of cancer survivors suffering from CIPN can be anticipated to expand in the foreseeable future, making its management an increasingly important issue.

Rehabilitation may play an important role in the recovery process and in improving daily functioning in patients with CIPN. Rehabilitation training can be offered to cancer patients during, as well as after completion of chemotherapy treatment. The optimal timing and intensity of such programs are currently being investigated in a large randomized clinical trial in the Netherlands. ${ }^{14}$ Interest in the role of exercise and physical rehabilitation programs as a symptomatic treatment for patients with CIPN has been increasing. ${ }^{1,15-20}$ Tofthagen et al. proposed a conceptual model in which exercise, by increasing mitochondrial energy production and blood flow to the peripheral nervous system, may result in fewer neuropathic symptoms. ${ }^{15}$ Wonders et al. argued that exercise could be an effective strategy in preserving neurological function following chemotherapy, suggesting a possible analogy in the pathogenesis of diabetic peripheral neuropathy (DPN) and CIPN through SERCA2a expression and ErbB2 activation. ${ }^{20}$ However, to this date these theories regarding the possible beneficial effects of exercise programs on gait, balance, mobility and muscle strength in cancer survivors with CIPN remain to be investigated in clinical trials in this particular subgroup of cancer patients. This article aims to consider the theoretical role of one particular modality, namely whole-body vibration (WBV), as a beneficial component of rehabilitation programs for CIPN patients.

Nowadays, vibrating platforms for WBV training are practically available to anyone who holds a gym license. Such vibrating platforms are powered by an engine, and can vibrate mechanically with various frequencies and amplitudes. ${ }^{21}$ The mechanical oscillations cause energy to be transferred from the vibrating platform to the lower extremities. Consequently, the human body standing on the vibrating platform acts as a resonator to the mechanical oscillations. ${ }^{22}$ Partly supported by scientific literature, manufacturers promise health effects, varying from a higher bone-density, ${ }^{23,24}$ improved muscle power, ${ }^{24,25}$ increased joint stability, ${ }^{26}$ oxygen blood flow, ${ }^{27}$ and $\mathrm{GH}$ hormone production ${ }^{25,28}$ to a more effective glucose uptake. ${ }^{29}$ The available literature indeed shows some controversial results and some of the study designs can be rightfully criticized. Nevertheless, as also argued in a comprehensive review by Rittweger, ${ }^{22}$ there seems to be a growing evidence base justifying the interest in the possible advantages of WBV in both the fields of sports and medicine. In addition to vertical vibration platforms, socalled side-alternating vibrating platforms exist. In side-alternating vibration, the left and right side of the platform are raised alternately, as opposed to conventional vertical vibration in which both sides are raised simultaneously. Using side-alternating vibration would supposedly result in more effective training of the back muscles ${ }^{30}$ and in a smaller vibration-load being transferred to the trunk. ${ }^{22}$

As it will be described in more detail further on, from a theoretical point of view WBV seems an appealing training modality in the rehabilitation of CIPN. Additionally, it would be an easily implementable addition to current rehabilitation programs for cancer survivors. Since there have been no studies researching any rehabilitation options for patients with CIPN to this date, this review will focus on the available evidence for effects of WBV on respectively muscle strength, balance and pain in patients with peripheral neuropathies that are not specifically chemotherapy-induced, but of any etiology.

\section{Methods of research}

The electronic database PubMed was systematically searched by the first author (AV) for articles describing clinical trials on WBV interven- tions, aimed at improving strength, balance and pain for patients with peripheral neuropathies, which were published until $5^{\text {th }}$ June 2014. Using a broad search strategy, a variety of search terms for the disorder peripheral neuropathy was combined (AND) with a broad selection of search terms for the intervention whole-body vibration and subsequently combined with search terms for the outcomes, respectively strength, balance and pain.

Inclusion criteria were: i) studies published in English; ii) studies involving adult human subjects with a peripheral neuropathy; iii) studies evaluating the effect of WBV as a therapeutic intervention; and iv) studies reporting findings for at least one of the following outcomes: strength, balance or pain.

The titles and abstracts retrieved from the initial database search were screened by the first author, using the inclusion criteria as described. Related citations in PubMed and reference lists of selected articles were searched for additional studies. Methodological quality of included studies was assessed independently by first (AV) and second author (HV), using the physiotherapy evidence database (PEDro) scale for clinical trials. The PEDro scale is an 11-item scale used to rate randomized controlled trials, designed by the Centre of Evidence-Based Physiotherapy. It covers all assessment criteria included in the Jadad scale and Delphi list. ${ }^{31}$ Results were compared and differences were resolved by discussion. Final levels of evidence and grades of recommendations were established using the grading system of the Scottish Intercollegiate Guidelines Network. ${ }^{32}$

\section{Results}

The search generated 91 results for the outcome strength, 63 results for the outcome balance and 351 results for the outcome pain. These results yielded a total of 5 article ${ }^{33-37}$ that met the inclusion criteria. Four studies ${ }^{33-35,37}$ worked with patients suffering from DPN and one study $^{36}$ with HIV (human immunodeficiency virus) -positive patients suffering from painful distal symmetrical polyneuropathy (DSPN). Two article ${ }^{35,37}$ described the effect of WBV on both strength and balance, and three articles ${ }^{33,34,36}$ described the effect of WBV on pain. The overall methodological quality was low, with four studies ${ }^{33,34,36,37}$ scoring 0 4 on the PEDro scale and one study ${ }^{35}$ scoring 7 (Table 1). ${ }^{33-37}$

Two studies ${ }^{33,34}$ in subjects with DPN found a beneficial effect of WBV on neuropathic pain, but another study ${ }^{36}$ failed to find the same effect for pain-relief in HIV-associated DSPN. One study ${ }^{35}$ found significant improvements in both muscle strength and balance, while another study ${ }^{37}$ found improvements only in some, but not all, of the applied tests to measure muscle strength and balance. The main findings of the included studies are summarized in Table $2{ }^{33-37}$

The final levels of evidence and grades of recommendation for the effects of WBV on pain, balance and muscle strength in patients with peripheral neuropathies are summarized in Table 3, using the grading system of the Scottish Intercollegiate Guidelines Network. ${ }^{32}$

\section{Discussion}

As indicated before, CIPN still comprise a major challenge as a doselimiting factor in cancer treatment, for which as of yet no adequate method of prevention or cure has been identified. The results of this literature search suggest a low level of evidence for a positive effect of WBV on muscle strength and balance and for no effect of WBV on neuropathic pain in patients with peripheral neuropathies. However, the body of evidence is small and of low methodological quality. Additionally, none of the studies includes outcome measures such as 
Table 1. Methodological quality of included studies.

\begin{tabular}{|c|c|c|c|c|c|}
\hline Internal validity - Statistical reporting & Hong et al. ${ }^{33}$ & Kessler et al. ${ }^{34}$ & Lee et $a l .35$ & Paice et $a l .{ }^{36}$ & Yoosefinejad et al. ${ }^{37}$ \\
\hline Random allocation & - & - & + & + & - \\
\hline Concealed allocation & - & - & + & - & - \\
\hline Baseline similarity & - & - & + & - & - \\
\hline Subject blinding & - & - & - & + & - \\
\hline Therapist blinding & - & - & - & - & - \\
\hline Assessor blinding & - & - & + & + & - \\
\hline Follow-up $>85 \%$ & + & - & + & - & + \\
\hline Intention-to-treat analysis & - & - & - & - & - \\
\hline Between-group comparisons & - & - & + & + & - \\
\hline Point measures and measures of variability & - & - & + & - & + \\
\hline PEDro score & 1 & 0 & 7 & 4 & 2 \\
\hline Eligibility criteria & - & + & + & + & + \\
\hline
\end{tabular}

PEDro, physiotherapy evidence database.

Table 2. Summary of the main findings of included studies.

\begin{tabular}{|c|c|c|c|c|c|}
\hline & $\begin{array}{l}\text { Study } \\
\text { population }\end{array}$ & $\begin{array}{l}\text { Intervention } \\
\text { details }\end{array}$ & Outcome(s) & Main findings & $\begin{array}{l}\text { PEDro } \\
\text { score }\end{array}$ \\
\hline Hong et al. ${ }^{33}$ & DPN & $\begin{array}{l}\text { Exercise } \\
\text { Standing; knees slightly bent } \\
\text { WBV parameters } \\
\text { f: } 20 \mathrm{~Hz} \text {; u: ns }\end{array}$ & Pain & $\begin{array}{l}\text { A significant reduction of pain in the } \\
\text { VAS was found directly after application of WBV (P=ns) } \\
\text { Significant reduction of pain in the long-term was found in } \\
\text { both the VAS (P=ns) and in the NPS variables intensity, } \\
\text { sharpness, unpleasantness and deep pain ( } \mathrm{P}=\mathrm{ns} \text { ) }\end{array}$ & 1 \\
\hline Kessler et al. ${ }^{34}$ & DPN & $\begin{array}{l}\text { Exercise } \\
\text { Standing; knees bent at } 20^{\circ} \\
\text { to the vertical } \\
\text { WBV parameters } \\
\text { f: } 25 \mathrm{~Hz} \text {; u: } 5 \mathrm{~mm}\end{array}$ & Pain & $\begin{array}{l}\text { A significant acute reduction of pain was found in the VAS } \\
(\mathrm{P}=0.018) \\
\text { A significant chronic reduction of pain was observed } \\
\text { in both the VAS }(\mathrm{P}<0.001) \text { and in the NPS variables intense, } \\
\text { sharp, hot, sensitive, unpleasant and deep pain }(\mathrm{P}<0.05)\end{array}$ & 0 \\
\hline Lee et al. ${ }^{35}$ & DPN & $\begin{array}{l}\text { Exercise } \\
110^{\circ} \text { squatting position } \\
\text { WBV parameters } \\
\text { f: } 15-30 \mathrm{~Hz} \text {; u: } 1-3 \mathrm{~mm}\end{array}$ & $\begin{array}{l}\text { Strength, } \\
\text { balance }\end{array}$ & $\begin{array}{l}\text { Significant improvements were noted in the static balance, dynamic } \\
\text { balance and muscle strength in the WBV + balance exercise group, } \\
\text { compared to the balance exercise and control groups }(\mathrm{P}<0.05)\end{array}$ & 7 \\
\hline Paice et al..$^{36}$ & $\begin{array}{l}\text { HIV-associated } \\
\text { DSPN }\end{array}$ & $\begin{array}{l}\text { Exercise } \\
\text { None; subjects sitting } \\
\text { WBV parameters } \\
\text { f: } 58.3 \mathrm{~Hz} \text {; u: ns }\end{array}$ & Pain & $\begin{array}{l}\text { No statistically significant differences were found between the vibration } \\
\text { and sham groups with respect to percentage pain relief }(\mathrm{P}=0.19) \text { or the } \\
\text { pre- and post-treatment current-pain difference }(\mathrm{P}=0.92)\end{array}$ & \\
\hline Yoosefinejad et al. ${ }^{37}$ & DPN & $\begin{array}{l}\text { Exercise } \\
\text { Squatting with knees } \\
\text { in } 30^{\circ} \text { flexion } \\
\text { WBV parameters } \\
\mathrm{f}: 30 \mathrm{~Hz} ; \mathrm{u}: 2 \mathrm{~mm}\end{array}$ & $\begin{array}{l}\text { Strength, } \\
\text { balance }\end{array}$ & $\begin{array}{l}\text { Pre- and post-intervention difference strength was significant for m. tibialis } \\
\text { anterior strength }(\mathrm{P}=0.014) \text {, but not for } \mathrm{m} \text {. quadriceps and general strength } \\
(\mathrm{P}>0.05) \\
\text { Pre- and post-intervention difference in balance was significant } \\
\text { for TUGT }(\mathrm{P}=0.004) \text {, but not for ULS }(\mathrm{P}>0.05)\end{array}$ & is 2 \\
\hline
\end{tabular}

PEDro, physiotherapy evidence database; DPN, diabetic peripheral neuropathy; WBV, whole-body vibration f: frequency; u, amplitude; ns, not specified; VAS, visual analogue scale; NPS, neuropathic pain scale; HIV, human immunodeficiency virus; DSPN, distal symmetrical polyneuropathy; TUGT, timed up and go test; UST, unilateral stance test.

Table 3. Final levels of evidence and grades of recommendations for the effects of whole-body vibration on pain, balance and muscle strength in patients with peripheral neuropathies using the grading system of the Scottish Intercollegiate Guidelines Network.

\begin{tabular}{lccc} 
& Finding & Level of evidence & Grade of recommendation \\
Effect of WBV on pain & No effect & $2-$ & D \\
Effect of WBV on balance & Positive effect & $2+$ & $\mathrm{C}$ \\
\hline Effect of WBV on muscle strength & Positive effect & $2+$ & $\mathrm{C}$ \\
\hline
\end{tabular}

WBV, whole-body vibration. 
pinpricks or tingling, although these are symptoms which are most relevant in CIPN. Therefore, until more high-quality research on the subject is performed, it seems worthwhile to discuss some of the underlying theories that have led to considering WBV as a potentially interesting modality in the rehabilitation of peripheral neuropathies in general, and in CIPN specifically.

The first consideration concerns the potential effect of WBV on muscle strength. This potential effect is based on the theoretical assumption that vibration training induces chronic adaptive effects at a neuromuscular level. ${ }^{22,38}$ From a physiological point of view, these adaptive effects would derive from the large number of vibration-induced repetitive cycles of eccentric and concentric muscle contraction and from the specific adaptive effects of muscle spindles and mechanoreceptors. ${ }^{22}$ These specific reflex mechanisms, typically referred to as the tonic vibration reflex (TVR), seem to be involved in influencing muscle activity and motor-unit recruitment strategy during vibration, ${ }^{39}$ most likely with the aim of stabilizing the joint. In fact, it has been reported that the application of vibration directly to a muscle or a tendon stimulates Ia afferents inducing the TVR. ${ }^{37,40}$ Using vibrating tools during exercise has been suggested to elicit similar responses. ${ }^{41}$ TVR was also seen in muscle groups not directly stimulated with vibration. ${ }^{42}$ Furthermore, alterations in spindle sensitivity and gamma-drive have been observed during reinforcement maneuvers such as the Jendrassik maneuver. ${ }^{43}$ It follows that the elevated muscle activation and neuromuscular response observed with vibration in several studies ${ }^{44,45}$ may be the result not only of motor unit activation strategies, but also of alterations in spindle sensitivity, possibly facilitating muscle neuromuscular conditioning through specific training programs. ${ }^{38}$

Although less common than sensory symptoms, motor symptoms such as muscle weakness may occur in CIPN. Therefore, a modality intervening at the neuromuscular level may be well worth considering as a rehabilitative tool. High-intensity strength training programs have already been proven to be both safe and effective in cancer survivors, in the short term ${ }^{46,47}$ as well as up to a year after completion of the training program. ${ }^{48}$ Modalities such as WBV, which would potentially contribute to building muscle strength, could be a worthwhile addition to such programs.

The second consideration is the potential beneficial effect of WBV on balance in patients with CIPN, the research has shown that the risk of falling is increased in patients with CIPN ${ }^{19,49,50}$ Furthermore, it is also likely that cancer- and chemotherapy-related fatigue and consequent inactivity contribute to muscle wasting, further raising the risk of falling. ${ }^{15,49}$ Studies suggested that WBV improves balance in elderly, ${ }^{51-}$ ${ }^{54}$ even though there still seems to exist some ambiguity as to whether the effect of WBV is actually supplemental to traditional training programs. ${ }^{54}$ The effect of WBV on balance, being either direct or through increasing muscle strength, implies that vibration-training may be a worthwhile adjunct to balance training in cancer patients suffering from CIPN.

The third and final consideration is whether WBV could be of use in reducing neurologic pain in patients with CIPN, which is a common symptom and is described by patients as tingling, pinpricks, electrical shocks or stabbing. ${ }^{55}$ The supposed pain-reducing effect of WBV is twofold: both mechanisms, an acute and a chronic pain-reducing effect, have been described.

The acute pain-reducing effect of WBV in diabetic peripheral neuropathy could derive from peripheral mechanisms in agreement to the gate-control theory, in which the vibration-induced axonal signal temporarily inhibits the pain-signaling axons. ${ }^{34}$ This hypothesis shows some similarity with the spinal pathway possibly involved in the autogenic inhibition of gamma drive, as observed by Fromm et al. during muscle vibration. ${ }^{40}$ Another explanation for the acute pain-reducing effect would be that the areas processing respectively vibratory stimuli and pain, lay in close proximity of each other in the somatosensory cor- tex. ${ }^{34,56}$ The prevailing explanation for the chronic pain-reducing effect of WBV is based on the process of central sensitization. ${ }^{34}$ In chronic pain, there seems to be a lack of spinal inhibition of pain signaling, resulting in a hyperalgesic state. ${ }^{57} \mathrm{WBV}$ may compensate for this hyperalgesic state by generating presynaptic spinal inhibition and consequently reducing spinal excitability. ${ }^{58}$ Although so far the presynaptic inhibitory effect of WBV has been observed only in motor neurones, resulting e.g. in suppression of the Hoffmann reflex (H-wave) resulting from transcutaneous electrical stimulation after $40-\mathrm{Hz} \mathrm{WBV}^{59}$ it is plausible that in chronic pain a similar effect occurs for nociceptive stimuli in the spinal ganglia of sensory neurones. ${ }^{34}$

On a final note, the effect of the most commonly used neurotoxic agents in the treatment of cancer - being platinum-derivates, vinca alkaloids, taxanes and thalidomide - takes place at the level of the dorsal ganglia. ${ }^{2}$ Since the prevailing theory for the chronic pain-reducing effect of WBV is based on the process of presynaptic inhibition at the same level in the peripheral nervous system, ${ }^{34}$ it may be possible that WBV would have a favorable effect on neurologic pain in cancer patients with CIPN.

\section{Conclusions}

The results of this literature search suggest insufficient evidence to assess the effectiveness for the effects of WBV on neuropathic pain, muscle strength and balance in patients with peripheral neuropathies, as there is a clear lack of methodologically high-quality research on the subject. In order to further explore the potential role of WBV in the rehabilitation of CIPN, high-quality trials - based on possible mechanisms - in cancer survivors will need to be performed.

\section{References}

1. Grisold W, Cavaletti G, Windebank AJ. Peripheral neuropathies from chemotherapeutics and targeted agents: diagnosis, treatment, and prevention. Neuro Oncol 2012;14:iv45-54.

2. Park SB, Goldstein D, Krishnan AV, et al. Chemotherapy-induced peripheral neurotoxicity: a critical analysis. CA Cancer J Clin;63:419-37.

3. Visovsky C, Collins M, Abbott L, et al. Putting evidence into practice: evidence-based interventions for chemotherapy-induced peripheral neuropathy. Clin J Oncol Nurs 2007;11:901-13.

4. Wilkes G. Peripheral neuropathy related to chemotherapy. Semin Oncol Nurs 2007;23:162-73.

5. Ocean AJ, Vahdat LT. Chemotherapy-induced peripheral neuropathy: pathogenesis and emerging therapies. Support Care Cancer 2004;12:619-25.

6. Wolf S, Barton D, Kottschade L, et al. Chemotherapy-induced peripheral neuropathy: prevention and treatment strategies. Eur J Cancer 2008;44:1507-15.

7. Stubblefield MD, Burstein HJ, Burton AW, et al. NCCN task force report: management of neuropathy in cancer. J Natl Compr Canc Netw 2009;7:S1-S26; quiz S27-8.

8. Velasco R, Bruna J. [Chemotherapy-induced peripheral neuropathy: an unresolved issue]. Neurologia 2010;25:116-31.

9. Hausheer FH, Schilsky RL, Bain S, et al. Diagnosis, management, and evaluation of chemotherapy-induced peripheral neuropathy. Semin Oncol 2006;33:15-49.

10. Hershman DL, Lacchetti C, Dworkin RH, et al. Prevention and management of chemotherapy-induced peripheral neuropathy in survivors of adult cancers: American Society of Clinical Oncology 
Clinical Practice Guideline. J Clin Oncol 2014 [Epub ahead of print].

11. Driessen CM, de Kleine-Bolt KM, Vingerhoets AJ, et al. Assessing the impact of chemotherapy-induced peripheral neurotoxicity on the quality of life of cancer patients: the introduction of a new measure. Support Care Cancer 2012;20:877-81.

12. Mols F, Beijers T, Lemmens V, et al. Chemotherapy-induced neuropathy and its association with quality of life among 2- to 11-year colorectal cancer survivors: results from the population-based PROFILES registry. J Clin Oncol 2013;31:2699-707.

13. Tofthagen C. Patient perceptions associated with chemotherapyinduced peripheral neuropathy. Clin J Oncol Nurs 2010;14:E22-8.

14. Chinapaw MJ, Buffart LM, van Mechelen W, et al. Alpe d'HuZes cancer rehabilitation (A-CaRe) research: four randomized controlled exercise trials and economic evaluations in cancer patients and survivors. Int J Behav Med 2012;19:143-56.

15. Tofthagen C, Visovsky C, Berry DL. Strength and balance training for adults with peripheral neuropathy and high risk of fall: current evidence and implications for future research. Oncol Nurs Forum 2012;39:E416-24.

16. Stubblefield MD, McNeely ML, Alfano CM, Mayer DK. A prospective surveillance model for physical rehabilitation of women with breast cancer: chemotherapy-induced peripheral neuropathy. Cancer 2012;118:2250-60.

17. Harris SR, Schmitz KH, Campbell KL, McNeely ML. Clinical practice guidelines for breast cancer rehabilitation: syntheses of guideline recommendations and qualitative appraisals. Cancer 2012;118: 2312-24.

18. Speck RM, DeMichele A, Farrar JT, et al. Scope of symptoms and self-management strategies for chemotherapy-induced peripheral neuropathy in breast cancer patients. Support Care Cancer 2012;20:2433-9.

19. Hile ES, Fitzgerald GK, Studenski SA. Persistent mobility disability after neurotoxic chemotherapy. Phys Ther 2010;90:1649-57.

20. Wonders KY RB, Druru DG. Treatment strategies for chemotherapy-induced peripheral neuropathy: potential role of exercise. Oncol Rev 2010;4:117-25.

21. Power Plate North America, Inc. Body vibration apparatus. Patent: US 7563235 B2; 2009.

22. Rittweger J. Vibration as an exercise modality: how it may work, and what its potential might be. Eur J Appl Physiol 2010;108:877904.

23. Rubin C, Turner AS, Bain S, et al. Anabolism. Low mechanical signals strengthen long bones. Nature 2001;412:603-4.

24. Verschueren SM, Roelants M, Delecluse C, et al. Effect of 6-month whole body vibration training on hip density, muscle strength, and postural control in postmenopausal women: a randomized controlled pilot study. J Bone Miner Res 2004;19:352-9.

25. Bosco C, Iacovelli M, Tsarpela 0, et al. Hormonal responses to whole-body vibration in men. Eur J Appl Physiol 2000;81:449-54.

26. Melnyk M, Kofler B, Faist M, et al. Effect of a whole-body vibration session on knee stability. Int J Sports Med 2008;29:839-44.

27. Yamada E, Kusaka T, Miyamoto K, et al. Vastus lateralis oxygenation and blood volume measured by near-infrared spectroscopy during whole body vibration. Clin Physiol Funct Imaging 2005;25:2038.

28. Kvorning T, Bagger M, Caserotti P, Madsen K. Effects of vibration and resistance training on neuromuscular and hormonal measures. Eur J Appl Physiol 2006;96:615-25.

29. Di Loreto C, Ranchelli A, Lucidi P, et al. Effects of whole-body vibration exercise on the endocrine system of healthy men. J Endocrinol Invest 2004;27:323-7.

30. Galileo website: https:/www.galileo-training.com/

31. Maher CG, Sherrington C, Herbert RD, et al. Reliability of the
PEDro scale for rating quality of randomized controlled trials. Phys Ther 2003;83:713-21.

32. Harbour R, Miller J. A new system for grading recommendations in evidence based guidelines. BMJ 2001;323:334-6.

33. Hong J, Barnes M, Kessler N. Case study: use of vibration therapy in the treatment of diabetic peripheral small fiber neuropathy. $\mathrm{J}$ Bodyw Mov Ther 2013;17:235-8.

34. Kessler NJ, Hong J. Whole body vibration therapy for painful diabetic peripheral neuropathy: a pilot study. J Bodyw Mov Ther 2013;17:518-22.

35. Lee K, Lee S, Song C. Whole-body vibration training improves balance, muscle strength and glycosylated hemoglobin in elderly patients with diabetic neuropathy. Tohoku J Exp Med 2013;231:30514.

36. Paice JA, Shott S, Oldenburg FP, et al. Efficacy of a vibratory stimulus for the relief of HIV-associated neuropathic pain. Pain 2000;84:291-6.

37. Kordi Yoosefinejad A, Shadmehr A, Olyaei G, et al. The effectiveness of a single session of Whole-Body Vibration in improving the balance and the strength in type 2 diabetic patients with mild to moderate degree of peripheral neuropathy: a pilot study. J Bodyw Mov Ther 2014;18:82-6.

38. Nordlund MM, Thorstensson A. Strength training effects of wholebody vibration? Scand J Med Sci Sports 2007;17:12-7.

39. Bongiovanni LG, Hagbarth KE. Tonic vibration reflexes elicited during fatigue from maximal voluntary contractions in man. J Physiol 1990;423:1-14.

40. Fromm C, Noth J. Reflex responses of gamma motoneurones to vibration of the muscle they innervate. J Physiol 1976;256:117-36.

41. Bosco C CM, Colli R, Tihanyi J, et al. The influence of whole body vibration on jumping ability. Biol Sport 1998;15:157-64.

42. Yasuda T, Izumizaki M, Ishihara Y, et al. Effect of quadriceps contraction on upper limb position sense errors in humans. Eur J Appl Physiol 2006;96:511-6.

43. Ribot-Ciscar E, Rossi-Durand C, Roll JP. Increased muscle spindle sensitivity to movement during reinforcement manoeuvres in relaxed human subjects. J Physiol 2000;523:271-82.

44. Mischi M, Cardinale M. The effects of a $28-\mathrm{Hz}$ vibration on arm muscle activity during isometric exercise. Med Sci Sports Exerc 2009;41:645-53.

45. Cardinale M, Lim J. Electromyography activity of vastus lateralis muscle during whole-body vibrations of different frequencies. $\mathrm{J}$ Strength Cond Res 2003;17:621-4.

46. White CM, Pritchard J, Turner-Stokes L. Exercise for people with peripheral neuropathy. Cochrane Database Syst Rev 2004;4 :CD003904.

47. De Backer IC, Van Breda E, Vreugdenhil A, et al. High-intensity strength training improves quality of life in cancer survivors. Acta Oncol 2007;46:1143-51.

48. De Backer IC, Vreugdenhil G, Nijziel MR, et al. Long-term follow-up after cancer rehabilitation using high-intensity resistance training: persistent improvement of physical performance and quality of life. Br J Cancer 2008;99:30-6.

49. Tofthagen C, Overcash J, Kip K. Falls in persons with chemotherapy-induced peripheral neuropathy. Support Care Cancer 2012;20:583-9.

50. Wampler MA, Topp KS, Miaskowski C, et al. Quantitative and clinical description of postural instability in women with breast cancer treated with taxane chemotherapy. Arch Phys Med Rehabil 2007;88:1002-8.

51. Rogan S, Hilfiker R, Herren K, et al. Effects of whole-body vibration on postural control in elderly: a systematic review and meta-analysis. BMC Geriatr 2011;11:72.

52. Lam FM, Lau RW, Chung RC, Pang MY. The effect of whole body 
vibration on balance, mobility and falls in older adults: a systematic review and meta-analysis. Maturitas 2012;72:206-13.

53. Sitja-Rabert M, Rigau D, Fort Vanmeerghaeghe A, et al. Efficacy of whole body vibration exercise in older people: a systematic review. Disabil Rehabil 2012;34:883-93.

54. Merriman H, Jackson K. The effects of whole-body vibration training in aging adults: a systematic review. J Geriatr Phys Ther 2009;32:134-45.

55. Bouhassira D, Attal N, Fermanian J, et al. Development and validation of the Neuropathic Pain Symptom Inventory. Pain 2004;108:248-57.
56. Coghill RC, Talbot JD, Evans AC, et al. Distributed processing of pain and vibration by the human brain. J Neurosci 1994;14:4095-108.

57. Staud R, Vierck CJ, Cannon RL, et al. Abnormal sensitization and temporal summation of second pain (wind-up) in patients with fibromyalgia syndrome. Pain 2001;91:165-75.

58. Kipp K, Johnson ST, Doeringer JR, Hoffman MA. Spinal reflex excitability and homosynaptic depression after a bout of wholebody vibration. Muscle Nerve 2011;43:259-62.

59. Armstrong WJ, Nestle HN, Grinnell DC, et al. The acute effect of whole-body vibration on the hoffmann reflex. J Strength Cond Res 2008;22:471-6. 